\title{
Chebyshev type inequalities via generalized fractional conformable integrals
}

\author{
Kottakkaran Sooppy Nisar ${ }^{\text {* }}$, Gauhar Rahman² and Khaled Mehrez ${ }^{3}$
}

\author{
"Correspondence: \\ n.sooppy@psau.edu.sa; \\ ksnisar1@gmail.com \\ 1 Department of Mathematics, \\ College of Arts and Sciences, Prince \\ Sattam Bin Abdulaziz University, \\ Wadi Aldawasir, Saudi Arabia \\ Full list of author information is \\ available at the end of the article
}

\begin{abstract}
Our aim in this present paper is to establish several Chebyshev type inequalities involving generalized fractional conformable integral operator recently introduced by T.U. Khan and M.A. Khan (J. Comput. Appl. Math. 346:378-389, 2019). Also, we present Chebyshev type inequalities involving Riemann-Liouville type fractional conformable integral operators as a particular result of our main result.
\end{abstract}

MSC: Primary 26A33; secondary 26D10; 26D15; 90C23; 33B20

Keywords: Fractional integral; Generalized fractional conformable integral; Inequalities

\section{Introduction}

Fractional calculus is an extremely useful tool to carry out differentiation and integration of real or complex number orders. This subject has received great consideration from researchers and mathematicians throughout the last few decades. Beginning with the classical Riemann-Liouville fractional integral and derivative operators, a large number of fractional integral and derivative operators and their generalizations have been presented (see, e.g., [2-4]). Among a large number of the fractional integral operators developed, due to applications in many fields of sciences, the Riemann-Liouville fractional integral operator has been extensively investigated. Integrations with weight functions are utilized in numerous mathematical problems such as approximation theory, spectral analysis, statistical analysis, and the theory of statistical distributions. Recently Khan et al. [5] established certain inequalities of the Hermite-Hadamard type with applications. In [6], the authors established Ostrowski type inequalities involving conformable fractional integrals. In [7-10], the authors introduced Hermite-Hadamard-Fejer inequalities for conformable fractional integrals via preinvex functions, generalized inequalities via GGconvexity and GA-convexity, Hermite-Hadamard type inequalities via the Montgomery identity, and Hermite-Hadamard type inequalities pertaining conformable fractional integrals and their applications. Rahman et al. [11] established certain Chebyshev type inequalities involving fractional conformable integral operators. In [12], the authors introduced the Minkowski inequalities via generalized proportional fractional integral operators. Some new inequalities involving fractional conformable integrals are found in the work of Nisar et al. [13]. Also, many researchers (see, e.g., [14-23]) have established a

(c) The Author(s) 2019. This article is distributed under the terms of the Creative Commons Attribution 4.0 International License (http://creativecommons.org/licenses/by/4.0/), which permits unrestricted use, distribution, and reproduction in any medium, provided you give appropriate credit to the original author(s) and the source, provide a link to the Creative Commons license, and indicate if changes were made. 
variety of inequalities by employing Riemann-Liouville fractional integral and derivative operators and their generalizations.

Recall the Chebyshev inequality [24] for two integrable and synchronous functions $\Phi$ and $\Psi$ defined on $[a, b]$

$$
\frac{1}{b-a} \int_{a}^{b} \Phi(\theta) \Psi(\theta) d \theta \geq\left(\frac{1}{b-a} \int_{a}^{b} \Phi(\theta) d \theta\right)\left(\frac{1}{b-a} \int_{a}^{b} \Psi(\theta) d \theta\right) .
$$

The two functions $\Phi$ and $\Psi$ are said to be synchronous on $[a, b]$ if

$$
(\Phi(\zeta)-\Phi(\varrho))(\Psi(\zeta)-\Psi(\varrho)) \geq 0 \quad(\zeta, \varrho \in[a, b])
$$

In [25-28], various researchers studied and introduced various generalizations of inequality (1). In [29], Belarbi and Dahmani established the following results related to the Chebyshev inequalities via Riemann-Liouville fractional integral operators.

Theorem 1.1 Suppose that $\Phi$ and $\Psi$ are two synchronous functions defined on $[0, \infty)$. Then the following inequality holds for all $\theta>0, \xi>0$ :

$$
\mathcal{I}^{\xi}(\Phi \Psi)(\theta) \geq \frac{\Gamma(\xi+1)}{\theta^{\xi}} \mathcal{I}^{\xi} \Phi(\theta) \mathcal{I}^{\xi} \Psi(\theta) .
$$

Theorem 1.2 Suppose that $\Phi$ and $\Psi$ are two synchronous functions defined on $[0, \infty)$. Then the following inequality holds for all $\theta>0, \xi>0, \lambda>0$ :

$$
\frac{\theta^{\xi}}{\Gamma(\xi+1)} \mathcal{I}^{\lambda}(\Phi \Psi)(\theta)+\frac{\theta^{\lambda}}{\Gamma(\lambda+1)} \mathcal{I}^{\xi}(\Phi \Psi)(\theta) \geq \mathcal{I}^{\xi} \Phi(\theta) \mathcal{I}^{\lambda} \Psi(\theta)+\mathcal{I}^{\lambda} \Phi(\theta) \mathcal{I}^{\xi} \Psi(\theta) .
$$

Theorem 1.3 Suppose that $\left(\Phi_{j}\right)_{j=1, \ldots, n}$ are $n$ positive increasing functions defined on $[0, \infty)$. Then the following inequality holds for any $\theta>0, \xi>0$ :

$$
\mathcal{I}^{\xi}\left(\prod_{j=1}^{n} \Phi_{j}\right)(\theta) \geq\left(\mathcal{I}^{\xi}(1)\right)^{1-n} \prod_{i=1}^{n} \mathcal{I}^{\xi} \Phi_{j}(\theta) .
$$

Theorem 1.4 Suppose that $\Phi$ and $\Psi$ are two functions defined on $[0, \infty)$ such that $\Phi$ is increasing, $\Psi$ is differentiable, and there exists a real number $m:=\inf _{\theta \geq 0} g^{\prime}(\theta)$. Then the following inequality is valid for all $\theta>0, \xi>0$ :

$$
\mathcal{I}^{\xi}(\Phi \Psi)(\theta) \geq\left(\mathcal{I}^{\xi}(1)\right)^{-1} \mathcal{I}^{\xi} \Phi(\theta) \mathcal{I}^{\xi} \Psi(\theta)-\frac{m \theta}{\xi+1} \mathcal{I}^{\xi} \Phi(\theta)+m \mathcal{I}^{\xi}(\theta \Psi(\theta)) .
$$

The left and right generalized fractional conformable integral operators are presented respectively in [1] as follows:

$$
{ }_{\xi}^{\eta} \mathcal{I}_{a^{+}}^{\lambda} \Phi(\theta)=\frac{1}{\Gamma(\lambda)} \int_{a}^{\theta}\left(\frac{\theta^{\xi+\eta}-\tau^{\xi+\eta}}{\xi+\eta}\right)^{\lambda-1} \frac{\Phi(\tau)}{\tau^{1-\xi-\eta}} d \tau, \quad \theta>a,
$$

and

$$
{ }_{\xi}^{\eta} \mathcal{I}_{b^{-}}^{\lambda} \Phi(\theta)=\frac{1}{\Gamma(\lambda)} \int_{\theta}^{b}\left(\frac{\tau^{\xi+\eta}-\theta^{\xi+\eta}}{\xi+\eta}\right)^{\lambda-1} \frac{\Phi(\tau)}{\tau^{1-\xi-\eta}} d \tau, \quad \theta<b,
$$


where $\lambda \in \mathbb{C}, \Re(\lambda)>0, \xi \in(0,1], \eta \in \mathbb{R}, \xi+\eta \neq 0$, and $\Gamma$ is the well-known gamma function [30].

Remark 1 (i) If $\eta=0$ in (7) and (8), then we have the following Riemann-Liouville type fractional conformable integral operators:

$$
{ }_{\xi} \mathcal{I}_{a^{+}}^{\lambda} \Phi(\theta)=\frac{1}{\Gamma(\lambda)} \int_{a}^{\theta}\left(\frac{\theta^{\xi}-\tau^{\xi}}{\xi}\right)^{\lambda-1} \frac{\Phi(\tau)}{\tau^{1-\xi}} d \tau, \quad \theta>a,
$$

and

$$
\xi \mathcal{I}_{b^{-}}^{\lambda} \Phi(\theta)=\frac{1}{\Gamma(\lambda)} \int_{\theta}^{b}\left(\frac{\tau^{\xi}-\theta^{\xi}}{\xi}\right)^{\lambda-1} \frac{\Phi(\tau)}{\tau^{1-\xi}} d \tau, \quad \theta<b
$$

where $\lambda \in \mathbb{C}, \Re(\lambda)>0, \xi \in(0,1]$.

(ii) If $\xi=1$ in (9) and (10), then we obtain the following Riemann-Liouville fractional integral operators:

$$
\mathcal{I}_{a^{+}}^{\lambda} \Phi(\theta)=\frac{1}{\Gamma(\lambda)} \int_{a}^{\theta}(\theta-\tau)^{\lambda-1} \Phi(\tau) d \tau, \quad \theta>a,
$$

and

$$
\mathcal{I}_{b^{-}}^{\lambda} \Phi(\theta)=\frac{1}{\Gamma(\lambda)} \int_{\theta}^{b}(\tau-\theta)^{\lambda-1} \Phi(\tau) d \tau, \quad \theta<b,
$$

where $\lambda \in \mathbb{C}, \Re(\lambda)>0$.

In this paper, we consider the following one-sided fractional conformable integral operator for conformable integrable function $\Phi$ :

$$
{ }_{\xi}^{\eta} \mathcal{I}^{\lambda} \Phi(\theta)=\frac{1}{\Gamma(\lambda)} \int_{0}^{\theta}\left(\frac{\theta^{\xi+\eta}-\tau^{\xi+\eta}}{\xi+\eta}\right)^{\lambda-1} \frac{\Phi(\tau)}{\tau^{1-\xi-\eta}} d \tau
$$

where $\lambda \in \mathbb{C}, \Re(\lambda)>0, \xi \in(0,1], \eta \in \mathbb{R}$, and $\xi+\eta \neq 0$.

Remark 2 (i) When we let $\eta=0$, then (13) will lead to the following Riemann-Liouville type fractional conformable integral operator:

$$
\xi \mathcal{I}^{\lambda} \Phi(\theta)=\frac{1}{\Gamma(\lambda)} \int_{0}^{\theta}\left(\frac{\theta^{\xi}-\tau^{\xi}}{\xi}\right)^{\lambda-1} \frac{\Phi(\tau)}{\tau^{1-\xi}} d \tau .
$$

(ii) When we let $\eta=0$ and $\xi=1$, then (13) will lead to the following Riemann-Liouville fractional integral operator:

$$
\mathcal{I}^{\lambda} \Phi(\theta)=\frac{1}{\Gamma(\lambda)} \int_{0}^{\theta}(\theta-\tau)^{\lambda-1} \Phi(\tau) d \tau
$$

Our aim is to establish Chebyshev type inequalities with two synchronous functions for a new generalized integral. 


\section{Main results}

In this section, we present Chebyshev type inequalities involving generalized fractional conformable integral operator (13).

Theorem 2.1 Let $\Phi$ and $\Psi$ be two integrable functions which are synchronous on $[0, \infty)$.

Then

$$
\left({ }_{\xi}^{\eta} \mathcal{I}^{\lambda} \Phi \Psi\right)(\theta) \geq \frac{\Gamma(\lambda+1)(\xi+\eta)^{\lambda}}{\theta^{(\xi+\eta) \lambda}}\left({ }_{\xi}^{\eta} \mathcal{I}^{\lambda} \Phi\right)(\theta)\left({ }_{\xi}^{\eta} \mathcal{I}^{\lambda} \Psi\right)(\theta),
$$

where $\lambda \in \mathbb{C}, \Re(\lambda)>0, \xi \in(0,1], \eta \in \mathbb{R}, \xi+\eta \neq 0$, and $\Gamma$ is the gamma function.

Proof Since $\Phi$ and $\Psi$ are synchronous on $[0, \infty)$, we have

$$
(\Phi(\zeta)-\Phi(\varrho))(\Psi(\zeta)-\Psi(\varrho) \geq 0
$$

or equivalently

$$
\Phi(\zeta) \Psi(\zeta)+\Phi(\varrho) \Psi(\varrho) \geq \Phi(\zeta) \Psi(\varrho)+\Phi(\varrho) \Psi(\zeta)
$$

Multiplying both sides of (18) by $\frac{1}{\Gamma(\lambda) \zeta^{1-\xi-\eta}}\left(\frac{\theta^{\xi+\eta}-\zeta^{\xi+\eta}}{\xi+\eta}\right)^{\lambda-1}$ and integrating the resultant inequality with respect to $\zeta$ over $(0, \theta)$, we get

$$
\begin{aligned}
& \frac{1}{\Gamma(\lambda)} \int_{0}^{\theta}\left(\frac{\theta^{\xi+\eta}-\zeta^{\xi+\eta}}{\xi}\right)^{\lambda-1} \frac{\Phi(\zeta) \Psi(\zeta)}{\zeta^{1-\xi-\eta}} d \zeta+\frac{1}{\Gamma(\lambda)} \int_{0}^{\theta}\left(\frac{\theta^{\xi+\eta}-\zeta^{\xi+\eta}}{\xi+\eta}\right)^{\lambda-1} \frac{\Phi(\varrho) \Psi(\varrho)}{\zeta^{1-\xi-\eta}} d \zeta \\
& \geq \frac{1}{\Gamma(\lambda)} \int_{0}^{\theta}\left(\frac{\theta^{\xi+\eta}-\zeta^{\xi+\eta}}{\xi+\eta}\right)^{\lambda-1} \frac{\Phi(\zeta) \Psi(\varrho)}{\zeta^{1-\xi-\eta}} d \zeta \\
& \quad+\frac{1}{\Gamma(\lambda)} \int_{0}^{\theta}\left(\frac{\theta^{\xi+\eta}-\zeta^{\xi+\eta}}{\xi+\eta}\right)^{\lambda-1} \frac{\Phi(\varrho) \Psi(\zeta)}{\zeta^{1-\xi-\eta}} d \zeta .
\end{aligned}
$$

It follows that

$$
\begin{aligned}
& \left({ }_{\xi}^{\eta} \mathcal{I}^{\lambda} \Phi \Psi\right)(\theta)+\Phi(\varrho) \Psi(\varrho) \frac{1}{\Gamma(\lambda)} \int_{0}^{\theta}\left(\frac{\theta^{\xi+\eta}-\zeta^{\xi+\eta}}{\xi+\eta}\right)^{\lambda-1} \frac{d \zeta}{\zeta^{1-\xi-\eta}} \\
& \geq \Psi(\varrho)\left({ }_{\xi}^{\eta} \mathcal{I}^{\lambda} \Phi\right)(\theta)+\Phi(\varrho)\left({ }_{\xi}^{\eta} \mathcal{I}^{\lambda} \Psi\right)(\theta) .
\end{aligned}
$$

Thus, we obtain

$$
\left({ }_{\xi}^{\eta} \mathcal{I}^{\lambda} \Phi \Psi\right)(\theta)+\frac{\theta^{(\xi+\eta) \lambda}}{\Gamma(\lambda+1)(\xi+\eta)^{\lambda}} \Phi(\varrho) \Psi(\varrho) \geq \Psi(\varrho)\left({ }_{\xi}^{\eta} \mathcal{I}^{\lambda} \Phi\right)(\theta)+\Phi(\varrho)\left({ }_{\xi}^{\eta} \mathcal{I}^{\lambda} \Psi\right)(\theta),
$$

where

$$
\int_{0}^{\theta}\left(\frac{\theta^{\xi+\eta}-\zeta^{\xi+\eta}}{\xi+\eta}\right)^{\lambda-1} \frac{d \zeta}{\zeta^{1-\xi-\eta}}=\frac{\theta^{(\xi+\eta) \lambda}}{\lambda(\xi+\eta)^{\lambda}}
$$


Multiplying both sides of (19) by $\frac{1}{\Gamma(\lambda) \varrho^{1-\xi-\eta}}\left(\frac{\theta^{\xi+\eta}-\varrho^{\xi+\eta}}{\xi+\eta}\right)^{\lambda-1}$ and integrating the resultant identity with respect to $\varrho$ over $(0, \theta)$, we get

$$
\begin{aligned}
{ }_{\xi}^{\eta} \mathcal{I}^{\lambda} & \Psi \\
& \\
& +\frac{\theta^{(\xi+\eta) \lambda}}{\Gamma(\lambda)} \int_{0}^{\theta}\left(\frac{\theta^{\xi+\eta}-\varrho^{\xi+\eta}}{\xi+\eta}\right)^{\lambda-1} \frac{d \varrho}{\varrho^{1-\xi-\eta}} \\
\geq & \left({ }_{\xi}^{\eta} \mathcal{I}^{\lambda} \Phi\right)(\theta) \frac{1}{\Gamma(\lambda)} \int_{0}^{\theta}\left(\frac{\theta^{\xi+\eta}-\varrho^{\xi+\eta}}{\xi(\lambda)} \int_{0}^{\lambda-1} \frac{\Psi(\varrho)}{\xi+\eta} \frac{\theta^{\xi+\eta}-\varrho^{\xi+\eta}}{\varrho^{1-\xi-\eta}}\right)^{\lambda-1} \frac{\Phi(\varrho) \Psi(\varrho)}{\varrho^{1-\xi-\eta}} d \varrho \\
& +\left({ }_{\xi}^{\eta} \mathcal{I}^{\lambda} \Psi\right)(\theta) \frac{1}{\Gamma(\lambda)} \int_{0}^{\theta}\left(\frac{\theta^{\xi+\eta}-\varrho^{\xi+\eta}}{\xi+\eta}\right)^{\lambda-1} \frac{\Phi(\varrho)}{\varrho^{1-\xi-\eta}} d \varrho .
\end{aligned}
$$

It follows that

$$
\begin{gathered}
\frac{\theta^{(\xi+\eta) \lambda}}{\Gamma(\lambda+1)(\xi+\eta)^{\lambda}}\left({ }_{\xi}^{\eta} \mathcal{I}^{\lambda} \Phi \Psi\right)(\theta)+\frac{\theta^{(\xi+\eta) \lambda}}{\Gamma(\lambda+1)(\xi+\eta)^{\lambda}}\left({ }_{\xi}^{\eta} \mathcal{I}^{\lambda} \Phi \Psi\right)(\theta) \\
\geq\left({ }_{\xi}^{\eta} \mathcal{I}^{\lambda} \Phi\right)(\theta)\left({ }_{\xi}^{\eta} \mathcal{I}^{\lambda} \Psi\right)(\theta)+\left({ }_{\xi}^{\eta} \mathcal{I}^{\lambda} \Phi\right)(\theta)\left({ }_{\xi}^{\eta} \mathcal{I}^{\lambda} \Psi\right)(\theta),
\end{gathered}
$$

which completes the desired proof.

Corollary 1 Let $\Phi$ and $\Psi$ be two integrable functions which are synchronous on $[0, \infty)$.

Then

$$
\left({ }_{\xi} \mathcal{I}^{\lambda} \Phi \Psi\right)(\theta) \geq \frac{\Gamma(\lambda+1) \xi^{\lambda}}{\theta^{\xi \lambda}}\left({ }_{\xi} \mathcal{I}^{\lambda} g\right)(\theta)\left({ }_{\xi} \mathcal{I}^{\lambda} \Psi\right)(\theta),
$$

where $\xi \in(0,1], \lambda \in \mathbb{C}$, and $\Re(\lambda)>0$.

Proof If we take $\eta=0$ in Theorem 2.1, then we get the desired inequality involving Riemann-Liouville type conformable fractional integral.

Remark 3 Applying Theorem 2.1 for $\eta=0$ and $\xi=1$ will give Theorem 1.1 .

Theorem 2.2 Let $\Phi$ and $\Psi$ be two integrable functions which are synchronous on $[0, \infty)$. Then

$$
\begin{gathered}
\frac{\theta^{(\xi+\eta) \tau}}{\Gamma(\tau+1)(\xi+\eta)^{\tau}}\left({ }_{\xi}^{\eta} \mathcal{I}^{\lambda} \Phi \Psi\right)(\theta)+\frac{\theta^{(\xi+\eta) \lambda}}{\Gamma(\lambda+1)(\xi+\eta)^{\lambda}}\left({ }_{\xi}^{\eta} \mathcal{I}^{\tau} \Phi \Psi\right)(\theta) \\
\geq\left({ }_{\xi}^{\eta} \mathcal{I}^{\lambda} \Phi\right)(\theta)\left({ }_{\xi}^{\eta} \mathcal{I}^{\tau} \Psi\right)(\theta)+\left({ }_{\xi}^{\eta} \mathcal{I}^{\tau} \Phi\right)(\theta)\left({ }_{\xi}^{\eta} \mathcal{I}^{\lambda} \Psi\right)(\theta),
\end{gathered}
$$

where $\lambda, \tau \in \mathbb{C}, \Re(\lambda)>0, \Re(\tau)>0, \xi \in(0,1], \eta \in \mathbb{R}$, and $\xi+\eta \neq 0$.

Proof Multiplying both sides of (19) by $\frac{1}{\Gamma(\tau) \varrho^{1-\xi-\eta}}\left(\frac{\theta^{\xi+\eta}-\varrho^{\xi+\eta}}{\xi+\eta}\right)^{\tau-1}$ and integrating the resultant inequality with respect to $\varrho$ over $(0, \theta)$, we get

$$
\begin{aligned}
& \frac{\left({ }_{\xi}^{\eta} \mathcal{I}^{\lambda} \Phi \Psi\right)(\theta)}{\Gamma(\tau)} \int_{0}^{\theta}\left(\frac{\theta^{\xi+\eta}-\varrho^{\xi+\eta}}{\xi+\eta}\right)^{\tau-1} \frac{d \varrho}{\varrho^{1-\xi-\eta}} \\
& \quad+\frac{\theta^{(\xi+\eta) \lambda}}{\Gamma(\lambda+1)(\xi+\eta)^{\lambda}} \frac{1}{\Gamma(\tau)} \int_{0}^{\theta}\left(\frac{\theta^{\xi+\eta}-\varrho^{\xi+\eta}}{\xi+\eta}\right)^{\tau-1} \frac{\Phi(\varrho) \Psi(\varrho)}{\varrho^{1-\xi-\eta}} d \varrho
\end{aligned}
$$




$$
\begin{aligned}
\geq & \frac{\left.{ }_{\xi}^{\eta} \mathcal{I}^{\lambda} \Phi\right)(\theta)}{\Gamma(\tau)} \int_{0}^{\theta}\left(\frac{\theta^{\xi+\eta}-\varrho^{\xi+\eta}}{\xi+\eta}\right)^{\tau-1} \frac{\Psi(\varrho)}{\varrho^{1-\xi-\eta}} d \varrho \\
& +\frac{\left({ }_{\xi}^{\eta} \mathcal{I}^{\lambda} \Psi\right)(\theta)}{\Gamma(\tau)} \int_{0}^{\theta}\left(\frac{\theta^{\xi+\eta}-\varrho^{\xi+\eta}}{\xi+\eta}\right)^{\tau-1} \frac{\Phi(\varrho)}{\varrho^{1-\xi-\eta}} d \varrho .
\end{aligned}
$$

Therefore, we have

$$
\begin{aligned}
& \frac{\theta^{(\xi+\eta) \tau}}{\Gamma(\tau+1)(\xi+\eta)^{\tau}}\left({ }_{\xi}^{\eta} \mathcal{I}^{\lambda} \Phi \Psi\right)(\theta)+\frac{\theta^{(\xi+\eta) \lambda}}{\Gamma(\lambda+1)(\xi+\eta)^{\lambda}}\left({ }_{\xi}^{\eta} \mathcal{I}^{\tau} \Phi \Psi\right)(\theta) \\
& \geq\left({ }_{\xi}^{\eta} \mathcal{I}^{\lambda} \Phi\right)(\theta)\left({ }_{\xi}^{\eta} \mathcal{I}^{\tau} \Psi\right)(\theta)+\left({ }_{\xi}^{\eta} \mathcal{I}^{\tau} \Phi\right)(\theta)\left({ }_{\xi}^{\eta} \mathcal{I}^{\lambda} \Psi\right)(\theta),
\end{aligned}
$$

which completes the desired proof of Theorem 2.2.

Remark 4 If we consider $\tau=\lambda$ in Theorem 2.2, then we obtain Theorem 2.1.

Corollary 2 Suppose that $\Phi$ and $\Psi$ are two integrable functions which are synchronous on $[0, \infty)$. Then

$$
\begin{gathered}
\frac{\theta^{\xi \tau}}{\Gamma(\tau+1) \xi \tau}\left(\xi \mathcal{I}^{\lambda} \Phi \Psi\right)(\theta)+\frac{\theta^{\xi \lambda}}{\Gamma(\lambda+1) \xi^{\lambda}}\left({ }_{\xi} \mathcal{I}^{\tau} \Phi \Psi\right)(\theta) \\
\geq\left({ }_{\xi} \mathcal{I}^{\lambda} \Phi\right)(\theta)\left({ }_{\xi} \mathcal{I}^{\tau} \Psi\right)(\theta)+\left({ }_{\xi} \mathcal{I}^{\tau} \Phi\right)(\theta)\left({ }_{\xi} \mathcal{I}^{\lambda} \Psi\right)(\theta),
\end{gathered}
$$

where $\lambda, \tau \in \mathbb{C}, \Re(\lambda)>0, \Re(\tau)>0, \xi \in(0,1]$.

Proof If we take $\eta=0$ in Theorem 2.2, then we get the desired inequality involving Riemann-Liouville type fractional conformable integral operator.

Remark 5 If we consider $\eta=0$ and $\xi=12.2$, then we get Theorem 1.2.

Remark 6 The inequalities in Theorems 2.1 and 2.2 will be reversed if the functions are asynchronous on $[0, \infty)$.

Theorem 2.3 Let $\left(\Phi_{j}\right)_{j=1,2, \ldots, n}$ be $n$ positive increasing functions on $[0, \infty)$. Then, for $\theta>0$, $\xi \in(0,1], \eta \in \mathbb{R}, \lambda \in \mathbb{C}$, we have

$$
{ }_{\xi}^{\eta} \mathcal{I}^{\lambda}\left(\prod_{j=1}^{n} \Phi_{j}\right)(\theta) \geq\left({ }_{\xi}^{n} \mathcal{I}^{\lambda}(1)\right)^{1-n} \prod_{j=1}^{n}\left({ }_{\xi}^{\eta} \mathcal{I}^{\lambda} \Phi_{j}\right)(\theta) .
$$

Proof To prove this theorem, we apply induction on $n$. Obviously, for $n=1$, we have

$$
{ }_{\xi}^{\eta} \mathcal{I}^{\lambda}\left(\Phi_{1}\right)(\theta) \geq{ }_{\xi}^{\eta} \mathcal{I}^{\lambda}\left(\Phi_{1}\right)(\theta) \quad \text { for all } \theta>0
$$

holds. For $n=2$, since $\Phi_{1}$ and $\Phi_{2}$ are positive and increasing functions, therefore we have

$$
\left(\Phi_{1}(\theta)-\Phi_{1}(y)\right)\left(\Phi_{2}(\theta)-\Phi_{2}(y)\right) \geq 0
$$

Hence, by applying Theorem 2.1, we obtain

$$
{ }_{\xi}^{\eta} \mathcal{I}^{\lambda}\left(\Phi_{1} \Phi_{2}\right)(\theta) \geq\left({ }_{\xi}^{\eta} \mathcal{I}^{\lambda}(1)\right)^{-1 \eta} \mathcal{I}^{\lambda}\left(\Phi_{1}\right)(\theta){ }_{\xi}^{\eta} \mathcal{I}^{\lambda}\left(\Phi_{2}\right)(\theta) \quad \text { for all } \theta>0 .
$$


Now, assume that by induction hypothesis

$$
{ }_{\xi}^{\eta} \mathcal{I}^{\lambda}\left(\prod_{j=1}^{n-1} \Phi_{j}\right)(\theta) \geq\left({ }_{\xi}^{\eta} \mathcal{I}^{\lambda}(1)\right)^{2-n} \prod_{j=1}^{n-1}\left({ }_{\xi}^{\eta} \mathcal{I}^{\lambda} \Phi_{j}\right)(\theta), \quad \theta>0
$$

Since $\Phi_{j} ; j=1,2, \ldots, n$, are positive increasing functions on $\mathbb{R}^{+}$, therefore $g:=\prod_{j=1}^{n-1} \Phi_{j}$ is increasing on $\mathbb{R}^{+}$. Let $h:=\Phi_{n}$. Applying Theorem 2.1 to the functions $\Phi$ and $\Psi$, we have

$$
{ }_{\xi}^{\eta} \mathcal{I}^{\lambda}\left(\prod_{j=1}^{n} \Phi_{j}\right)(\theta)={ }_{\xi}^{\eta} \mathcal{I}^{\lambda}(\Phi \Psi)(\theta) \geq\left({ }_{\xi}^{\eta} \mathcal{I}^{\lambda}(1)\right)^{-1}{ }_{\xi}^{\eta} \mathcal{I}^{\lambda}\left(\prod_{j=1}^{n-1} \Phi_{j}\right)(\theta)\left({ }_{\xi}^{\eta} \mathcal{I}^{\lambda} \Phi_{n}\right)(\theta)
$$

By using (24), we obtain

$$
{ }_{\xi}^{\eta} \mathcal{I}^{\lambda}\left(\prod_{j=1}^{n} \Phi_{j}\right)(\theta) \geq\left({ }_{\xi}^{\eta} \mathcal{I}^{\lambda}(1)\right)^{-1}\left({ }_{\xi}^{\eta} \mathcal{I}^{\lambda}(1)\right)^{2-n}\left({ }_{\xi}^{\eta} \mathcal{I}^{\lambda} \prod_{j=1}^{n-1} \Phi_{j}\right)(\theta)\left({ }_{\xi}^{\eta} \mathcal{I}^{\lambda} \Phi_{n}\right)(\theta)
$$

which completes the desired proof.

Corollary 3 Suppose that $\left(\Phi_{j}\right)_{j=1,2, \ldots, n}$ are $n$ positive increasing functions on $[0, \infty)$. Then, for $\theta>0, \xi \in(0,1], \lambda \in \mathbb{C}$, and $\Re(\lambda)>0$, we have

$$
{ }_{\xi} \mathcal{I}^{\lambda}\left(\prod_{j=1}^{n} \Phi_{j}\right)(\theta) \geq\left({ }_{\xi} \mathcal{I}^{\lambda}(1)\right)^{n-1} \prod_{j=1}^{n}\left({ }_{\xi} \mathcal{I}^{\lambda} \Phi_{j}\right)(\theta)
$$

Proof If we let $\eta=0$ in Theorem 2.3, then we get the desired corollary involving RiemannLiouville type fractional conformable integral operator.

Remark 7 If we let $\eta=0$ and $\xi=1$ in Theorem 2.3, we get Theorem 1.3.

Theorem 2.4 Let $\xi \in(0,1], \eta \in \mathbb{R}, \lambda \in \mathbb{C}, \mathfrak{R}(\lambda)>0$, and $\xi+\eta \neq 0$. Also, let two functions $\Phi, \Psi: \mathbb{R}_{0}^{+} \rightarrow \mathbb{R}$ such that $\Phi$ is increasing and $\Psi$ is differentiable with $\Psi^{\prime}$ bounded below, and let $m=\inf _{\theta \in \mathbb{R}_{0}^{+}} \Psi^{\prime}(\theta)$. Then

$$
\begin{aligned}
\left({ }_{\xi}^{\eta} \mathcal{I}^{\lambda} \Phi \Psi\right)(\theta) \geq & \frac{\Gamma(\lambda+1)(\xi+\eta)^{\lambda}}{\theta^{(\xi+\eta) \lambda}}\left({ }_{\xi}^{\eta} \mathcal{I}^{\lambda} \Phi\right)(\theta)\left({ }_{\xi}^{\eta} \mathcal{I}^{\lambda} \Psi\right)(\theta) \\
& -\frac{m x}{(\lambda+1)}\left({ }_{\xi}^{\eta} \mathcal{I}^{\lambda} \Phi\right)(\theta)+m\left({ }_{\xi}^{\eta} \mathcal{I}^{\lambda} i \Phi\right)(\theta),
\end{aligned}
$$

where $i(\theta)$ is the identity function.

Proof Let $\Psi(\theta)=\Psi(\theta)-m \theta^{\xi+\eta}$. We find that $\Psi$ is differentiable and increasing on $\mathbb{R}_{0}^{+}$. As in the process of Theorem 2.3 , for clarity, let $p(\theta):=m \theta^{\xi+\eta}$, we obtain

$$
\begin{aligned}
\left({ }_{\xi}^{\eta} \mathcal{I}^{\lambda} g(h-p)\right)(\theta) \geq & \frac{\Gamma(\lambda+1)(\xi+\eta)^{\lambda}}{\theta^{(\xi+\eta) \lambda}}\left({ }_{\xi}^{\eta} \mathcal{I}^{\lambda} \Phi\right)(\theta)\left({ }_{\xi}^{\eta} \mathcal{I}^{\lambda}(h-p)\right)(\theta) \\
= & \frac{\Gamma(\lambda+1)(\xi+\eta)^{\lambda}}{\theta^{(\xi+\eta) \lambda}}\left({ }_{\xi}^{\eta} \mathcal{I}^{\lambda} \Phi\right)(\theta)\left({ }_{\xi}^{\eta} \mathcal{I}^{\lambda} \Psi\right)(\theta) \\
& -\frac{\Gamma(\lambda+1)(\xi+\eta)^{\lambda}}{\theta^{(\xi+\eta) \lambda}}\left({ }_{\xi}^{\eta} \mathcal{I}^{\lambda} \Phi\right)(\theta)\left({ }_{\xi}^{\eta} \mathcal{I}^{\lambda} p\right)(\theta) .
\end{aligned}
$$


We have

$$
\left({ }_{\xi}^{\eta} \mathcal{I}^{\lambda} g(h-p)\right)(\theta)=\left({ }_{\xi}^{\eta} \mathcal{I}^{\lambda} \Phi \Psi\right)(\theta)-m\left({ }_{\xi}^{\eta} \mathcal{I}^{\lambda} i \Phi\right)(\theta)
$$

and

$$
\left({ }_{\xi}^{\eta} \mathcal{I}^{\lambda} p\right)(\theta)=\frac{m \theta^{(\xi+\eta) \lambda+1}}{\Gamma(\lambda+2)(\xi+\eta)^{\lambda}} .
$$

Finally using (29) and (30) in (28), we obtain the desired result.

Corollary 4 Let $\xi \in(0,1], \lambda \in \mathbb{C}$, and $\Re(\lambda)>0$. Also, let two functions $\Phi, \Psi: \mathbb{R}_{0}^{+} \rightarrow \mathbb{R}$ such that $\Phi$ is increasing and $\Psi$ is differentiable with $\Psi^{\prime}$ bounded below, and let $m=$ $\inf _{\theta \in \mathbb{R}_{0}^{+}} \Psi^{\prime}(\theta)$. Then

$$
\begin{aligned}
\left({ }_{\xi} \mathcal{I}^{\lambda} \Phi \Psi\right)(\theta) \geq & \frac{\Gamma(\lambda+1) \xi^{\lambda}}{\theta^{\xi \lambda}}\left({ }_{\xi} \mathcal{I}^{\lambda} \Phi\right)(\theta)\left({ }_{\xi} \mathcal{I}^{\lambda} \Psi\right)(\theta) \\
& -\frac{m x}{(\lambda+1)}\left({ }^{\lambda} \mathcal{I}^{\lambda} \Phi\right)(\theta)+m\left({ }_{\xi} \mathcal{I}^{\lambda} i \Psi\right)(\theta),
\end{aligned}
$$

where $i(\theta)$ is the identity function.

Proof If we take $\eta=0$ in Theorem 2.4, then we get the desired Corollary 4, which involves Riemann-Liouville type fractional conformable integral operator.

Remark 8 If we let $\xi=1$ and $\eta=0$ in Theorem 2.4, then we obtain Theorem 1.4.

\section{Concluding remarks}

Several Chebyshev type inequalities involving generalized conformable fractional integral operators are introduced in this paper. Also, we presented some particular results which involve Riemann-Liouville type conformable fractional integral operator. We observed that if we let $\xi=1$ and $\eta=0$, then the inequalities obtained in this paper will reduce to the inequalities obtained earlier by Belarbi and Dahmani [29].

Acknowledgements

This project was supported by the Deanship of Scientific Research at Prince Sattam Bin Abdulaziz University under the research project \# 2019/01/10384.

Funding

Not applicable.

Availability of data and materials

Not applicable.

Competing interests

The authors declare that they have no competing interests.

Authors' contributions

All authors contributed equally. All authors read and approved the final manuscript.

\section{Author details}

${ }^{1}$ Department of Mathematics, College of Arts and Sciences, Prince Sattam Bin Abdulaziz University, Wadi Aldawasir, Saudi Arabia. ${ }^{2}$ Department of Mathematics, Shaheed Benazir Bhutto University, Sheringal, Pakistan. ${ }^{3}$ Department of Mathematics, Issat Kasserine, University of Kairouan, Kairouan, Tunisia. 


\section{Publisher's Note}

Springer Nature remains neutral with regard to jurisdictional claims in published maps and institutional affiliations.

\section{Received: 16 April 2019 Accepted: 2 September 2019 Published online: 11 September 2019}

\section{References}

1. Khan, T.U., Khan, M.A.: Generalized conformable fractional integral operators. J. Comput. Appl. Math. 346, 378-389 (2019). https://doi.org/10.1016/j.cam.2018.07.018

2. Kilbas, A.A., Srivastava, H.M., Truhillo, J.J.: Theory and Applications of Fractional Differential Equations. Elsevier, Amsterdam (2006)

3. Podlubny, l.: Fractional Differential Equations. Academic Press, London (1999)

4. Samko, S.G., Kilbas, A.A., Marichev, O.I.: Fractional Integrals and Derivatives: Theory and Applications. Gordon \& Breach, Reading (1993)

5. Khan, M.A., Khurshid, Y., Dragomir, S.S., Ullah, R.: Inequalities of the Hermite-Hadamard type with applications. Punjab Univ. J. Math. 50(3), 1-12 (2018)

6. Khan, M.A., Begum, S., Khurshid, Y., Chu, Y.M.: Ostrowski type inequalities involving conformable fractional integrals. J. Inequal. Appl. 2018, 70, 1-14 (2018)

7. Khurshid, Y., Adil Khan, M., Chu, Y.M.: Hermite-Hadamard-Fejer inequalities for conformable fractional integrals via preinvex functions. J. Funct. Spaces 2019, 1-9 Article ID 3146210 (2019)

8. Khurshid, Y., Khan, M.A., Chu, Y.M.: Generalized inequalities via GG-convexity and GA-convexity. J. Funct. Spaces 2019, Article ID 6926107, 1-8 (2019)

9. Khan, M.A., Khurshid, Y., Chu, Y.M.: Hermite-Hadamard type inequalities via the Montgomery identity. Commun. Math. Appl. 10(1), 85-97 (2019)

10. Iqbal, A., Khan, M.A., Ullah, S., Kashuri, A., Chu, Y.M.: Hermite-Hadamard type inequalities pertaining conformable fractional integrals and their applications. AIP Adv. 8, 075101, 1-18 (2018)

11. Rahman, G., Ullah, Z., Khan, A., Set, E., Nisar, K.S.: Certain Chebyshev type inequalities involving fractional conformable integral operators. Mathematics 7, 364 (2019). https://doi.org/10.3390/math7040364

12. Rahman, G., Khan, A., Abdeljawad, T., Nisar, K.S.: The Minkowski inequalities via generalized proportional fractional integral operators. Adv. Differ. Equ. 2019, 287 (2019). https://doi.org/10.1186/s13662-019-2229-7

13. Nisar, K.S., Tassaddiq, A., Rahman, G., Khan, A.: Some inequalities via fractional conformable integral operators. J. Inequal. Appl. 2019, 217 (2019)

14. Dahmani, Z., Tabharit, L.: On weighted Gruss type inequalities via fractional integration. J. Adv. Res. Pure Math. 2, 31-38 (2010)

15. Dahmani, Z.: New inequalities in fractional integrals. Int. J. Nonlinear Sci. 9(4), 493-497 (2010)

16. Kiryakova, V:: Generalized Fractional Calculus and Applications. Pitman Res. Notes Math. Ser., vol. 301. Longman, New York (1994)

17. Katrakhov, V.V., Sitnik, S.M.: The transmutation method and boundary-value problems for singular elliptic equations. Contemp. Math Fundam. Dir. 64, 211-426 (2018)

18. Ntouyas, K.S., Agarwal, P., Tariboon, J.: On Polya-Szego and Chebyshev types inequalities involving the Riemann-Liouville fractional integral operators. J. Math. Inequal. 10(2), 491-504 (2016)

19. Nisar, K.S., Qi, F., Rahman, G., Mubeen, S., Arshad, M.: Some inequalities involving the extended gamma function and the Kummer confluent hypergeometric k-function. J. Inequal. Appl. 2018, 135 (2018)

20. Nisar, K.S., Rahman, G., Choi, J., Mubeen, S., Arshad, M.: Certain Gronwall type inequalities associated with Riemann-Liouville k- and Hadamard k-fractional derivatives and their applications. East Asian Math. J. 34(3), 249-263 (2018)

21. Sarikaya, M.Z., Dahmani, Z., Kiris, M.E., Ahmad, F.: (k,s)-Riemann-Liouville fractional integral and applications. Hacet. J. Math. Stat. 45(1), 77-89 (2016)

22. Set, E., Tomar, M., Sarikaya, M.Z:: On generalized Grüss type inequalities for $k$-fractional integrals. Appl. Math. Comput. 269, 29-34 (2015)

23. Rahman, G., Nisar, K.S., Mubeen, S., Choi, J.: Certain inequalities involving the $(k, \rho)$-fractional integral operator. Far East J. Math. Sci.: FJMS 103(11), 1879-1888 (2018)

24. Chebyshev, P.L.: Sur les expressions approximatives des integrales definies par les autres prises entre les mmes limites. Proc. Math. Soc. Charkov 2, 93-98 (1882)

25. Rahman, G., Nisar, K.S., Qi, F.: Some new inequalities of the Gruss type for conformable fractional integrals. AIMS Math. 3(4), 575-583 (2018)

26. Qi, F., Rahman, G., Hussain, S.M., Du, W.S., Nisar, K.S.: Some inequalities of Čebyšev type for conformable k-fractional integral operators. Symmetry 10, 614 (2018). https://doi.org/10.3390/sym10110614

27. Özdemir, M.E., Set, E., Akdemir, A.O., Sarkaya, M.Z.: Some new Chebyshev type inequalities for functions whose derivatives belong to $L_{p}$ spaces. Afr. Math. 26, 1609-1619 (2015)

28. Set, E., Dahmani, Z., Mumcu, I.: New extensions of Chebyshev type inequalities using generalized Katugampola integrals via Polya-Szeg inequality. Int. J. Optim. Control Theor. Appl. 8(2), 137-144 (2018)

29. Belarbi, S., Dahmani, Z:: On some new fractional integral inequalities. J. Inequal. Pure Appl. Math. 10(3), Article 86, 5 pp. (2009)

30. Srivastava, H.M., Choi, J.: Zeta and q-Zeta Functions and Associated Series and Integrals. Elsevier, Amsterdam (2012) 